\title{
Extended Reality (XR) Remote Research: a Survey of Drawbacks and Opportunities
}

\author{
Jack Ratcliffe ${ }^{*}$ \\ Queen Mary, University of London \\ London, UK
}

\author{
Francesco Soave* \\ Queen Mary, University of London \\ London, UK
}

\author{
Nick Bryan-Kinns \\ Queen Mary University of London \\ London, UK
}

\author{
Laurissa Tokarchuk \\ Queen Mary University of London \\ London, UK
}

\begin{abstract}
Extended Reality (XR) technology - such as virtual and augmented reality - is now widely used in Human Computer Interaction (HCI), social science and psychology experimentation. However, these experiments are predominantly deployed in-lab with a co-present researcher. Remote experiments, without co-present researchers, have not flourished, despite the success of remote approaches for non-XR investigations. This paper summarises findings from a 30-item survey of $46 \mathrm{XR}$ researchers to understand perceived limitations and benefits of remote XR experimentation. Our thematic analysis identifies concerns common with non-XR remote research, such as participant recruitment, as well as XR-specific issues, including safety and hardware variability. We identify potential positive affordances of XR technology, including leveraging data collection functionalities builtin to HMDs (e.g. hand, gaze tracking) and the portability and reproducibility of an experimental setting. We suggest that XR technology could be conceptualised as an interactive technology and a capable data-collection device suited for remote experimentation.
\end{abstract}

\section{CCS CONCEPTS}

- Human-centered computing $\rightarrow$ Mixed / augmented reality; Virtual reality.

\section{KEYWORDS}

Extended Reality, Virtual Reality, Augmented Reality, literature review, expert interviews

\section{ACM Reference Format:}

Jack Ratcliffe, Francesco Soave, Nick Bryan-Kinns, Laurissa Tokarchuk, and Ildar Farkhatdinov. 2021. Extended Reality (XR) Remote Research: a Survey of Drawbacks and Opportunities. In CHI Conference on Human Factors in Computing Systems (CHI '21), May 8-13, 2021, Yokohama, Japan. ACM, New York, NY, USA, 13 pages. https://doi.org/10.1145/3411764.3445170

${ }^{*}$ Both authors contributed equally to this research.

Permission to make digital or hard copies of all or part of this work for personal or classroom use is granted without fee provided that copies are not made or distributed for profit or commercial advantage and that copies bear this notice and the full citation on the first page. Copyrights for components of this work owned by others than the author(s) must be honored. Abstracting with credit is permitted. To copy otherwise, or republish, to post on servers or to redistribute to lists, requires prior specific permission and/or a fee. Request permissions from permissions@acm.org.

CHI '21, May 8-13, 2021, Yokohama, Japan

(C) 2021 Copyright held by the owner/author(s). Publication rights licensed to ACM. ACM ISBN 978-1-4503-8096-6/21/05 . .\$15.00

https://doi.org/10.1145/3411764.3445170

\author{
Ildar Farkhatdinov \\ Queen Mary University of London \\ London, UK
}

\section{INTRODUCTION}

Extended reality (XR) technology - such as virtual, augmented, and mixed reality - is increasingly being examined and utilised by researchers in the $\mathrm{HCI}$ and other research communities due to its potential for creative, social and psychological experiments [7]. Many of these studies take place in laboratories with the co-presence of the researcher and the participant [36]. The XR research community has been slow to embrace recruiting remote participants to take part in studies running outside of laboratories - a technique which has proven useful for non-XR HCI, social and psychological research [53][49]. However, the current Covid-19 pandemic has highlighted the importance and perhaps necessity of understanding and deploying remote recruitment methods within XR research.

There is also limited literature about remote $\mathrm{XR}$ research, although what reports exist suggest that the approach shows promise: data-collection is viable [67], results are similar to those found inlab [46] even when the participants are unsupervised [29], and recruiting is possible [40]. Researchers have also suggested using existing communities for these technologies, such as customisable social VR experiences, as combined platforms for recruitment and experimentation [60]. With the increasingly availability of consumer XR devices (estimates show five million high-end XR HMDs sold in 2020, raising to 43.5 million by 2025[73]), and health and safety concerns around in-lab experimentation, particularly for research involving head-mounted displays (HMDs), it seems an important time to understand the conceptions around remote research from researchers who use XR technologies.

This paper outlines the methodology and results from the first (that we are aware of) survey of XR researchers regarding remote $\mathrm{XR}$ research. The results have been derived from 46 respondents answering 30 questions regarding existing research practice. It offers three core contributions: (1) we summarise existing research on conducting remote XR experiments. (2) We provide an overview of the status quo, showing that many of the concerns regarding remote $\mathrm{XR}$ are those also applicable to other remote studies; and that the unique aspects of remote XR research could offer more benefits than drawbacks. (3) We set out recommendations for advancing remote XR research, and outline important questions that should be answered to create an evidence-backed experimentation process.

\section{LITERATURE}

We present a literature review of relevant publications on XR research, remote research and remote XR research. We use "XR" as the umbrella term for virtual reality (VR), augmented reality (AR) 
and mixed reality (MR) [39]. This space is also sometimes referred to as spatial or immersive computing.

The chapter is organised in three parts. First, we explore conventional XR experiments under 'normal' conditions (e.g. in laboratory andor directly supervised by the researcher). We then summarise existing literature on remote experiments in XR research. Finally, we report the main findings in previous publications on remote data collection and experimentation.

\subsection{Conventional XR experiments}

2.1.1 Experiment types and fields of interest. According to Suh and Prohpet's 2018 systematic literature review [71], XR experiments involving human participants can broadly be categorised into two groups: (1) studies about XR, and (2) studies about using XR. The first group focuses on the effects of XR system features on the user experience (e.g. if enhancing embodiment could affect presence outcomes [56]), whereas the second category examines how the use of an XR technology modifies a measurable user attribute (e.g. if leveraging XR embodiment could affect learning outcomes [55]). Across these categories there have been a variety of explorations on different subjects and from different academic fields. These include social psychological [7], including social facilitation-inhibition [28], conformity and social comparison [6], social identity [34]; neuroscience and neuropsychology [36], visual perception [78], multisensory integration [14], proxemics [61], spatial cognition [75], education and training [54], therapeutic applications [20], pain remediation [23], motor control [16], terror management [30] and media effects such as presence [4].

The theoretical approaches behind these studies are also disparate, including theories such as conceptual blending, cognitive load, constructive learning, experiential learning, flow, media richness, motivation, presence, situated cognition, the stimuli-organismresponse framework and the technology acceptance model [71].

2.1.2 Data collection, approaches and techniques. According to Suh and Prophet's meta-analysis, the majority of XR research explorations have been experiments (69\%) [71]. Other types of explorations include surveys (24\%), interviews (15\%) and case studies (9\%). These approaches have been used both alone and in combination with each other. Data collection methods are predominantly quantitative (78\%), although qualitative and mixed approaches are also used. Another systematic review of XR research (focused on higher education) [54] adds focus group discussion and observation as research methods, and presents two potential subcategories for experiments: mobile sensing and "interaction log in VR app", in which the XR application logs the user's activities and the researcher uses the resulting log for analysis.

The types of data logging found in XR experiments are much the same as those listed in Weibel's exploration of physiological measures in non-immersive virtual reality [77], with studies using skin conductance [79], heart rate [18], blood pressure [27], as well as electroencephalogram (EEG) [1]. Built-in inertial sensors that are integral to providing an XR experience, such as head and hand position for VR HMDs, have also been widely used for investigations, including posture assessment [9], head interaction tracking [80], gaze and loci of attention [52] and gesture recognition [31], while velocity change [76] has also been used in both VR and AR interventions.

2.1.3 Benefits of XR experiments. There are many suggested benefits to using XR technology as a research tool: it allows researchers to control the mundane-realism trade-off [2] and thus increase the extent to which an experiment is similar to situations encountered in everyday life without sacrificing experimental control [7]; to create powerful sensory illusions within a controlled environment (particularly in VR), such as illusions of self-motion and influence the proprioceptive sense [65]; improve replication [7] by making it easier to recreate entire experimental environments; and allow representative samples[7] to experience otherwise inaccessible environments, when paired with useful distribution and recruitment networks.

2.1.4 Challenges of XR experiments. Pan [47] explored some of the challenges facing experiments in virtual worlds, which continue to be relevant in immersive XR explorations. These include the challenge of ensuring the experimental design is relevant for each technology and subject area; ensuring a consistent feeling of selfembodiment to ensure engaged performance [35]; avoid uncanny valley, in which characters which look nearly-but-not-quite human are judged as uncanny and are aversive for participants [44]; simulation sickness and nausea during VR experiences[45]; cognitive load [72] which may harm participation results through overstimulation, particularly in VR [69] [41]; novelty effects of new technology interfering with results [15] [19]; and ethics, especially where experiences in VR could lead to changes in participants' behaviour and attitude in their real life [5] and create false memories [63].

\subsection{Remote XR experiments}

There has been little research into remote XR experimentation, particularly for VR and AR HMDs. By remote, we mean any experiment that takes place outside of a researcher-controlled setting. This is distinct from field or in-the-wild research, which is research "that seeks to understand new technology interventions in everyday living" [58], and so is dependent on user context. These definitions are somewhat challenged in the context of remote VR research, as for VR, remote and field/in-the-wild are often the same setting, as the location where VR is most used outside the lab is also where it is typically experienced (e.g. home users, playing at home [40]). For $\mathrm{AR}$, there is a greater distinction between remote, which refer to any AR outside of the controlled setting of the lab; and field/in-the-wild, which require a contextual deployment.

In terms of remote XR research outcomes, Mottelson and Hornbæk [46] directly compared in-lab and remote VR experiment results. They found that while the differences in performance between the in-lab and remote study were substantial, there were no significant differences between effects of experimental conditions. Similarly, Huber and Gajos explored uncompensated and unsupervised remote VR samples and were able to replicate key results from the original in-lab studies, although with smaller effect sizes [29]. Finally, Steed et al. showed that collecting data in the wild is feasible for virtual reality systems [67]. 
Ma et al. [40] is perhaps the first published research on recruiting remote participants for VR research. The study, published in 2018, used the Amazon Mechanical Turk (AMT) crowdsourcing platform, and received 439 submissions over a 13-day period, of which 242 were eligible. The participant demographics did not differ significantly from previously reported demographics of AMT populations in terms of age, gender, and household income. The notable difference was that the VR research had a higher percentage of U.S.-based workers compared to others. The study also provides insight into how remote XR studies take place: $98 \%$ of participants took part at home, in living rooms (24\%), bedrooms (18\%), and home offices $(18 \%)$. Participants were typically alone $(84 \%)$ or in the presence of one $(14 \%)$ or two other people (2\%). Participants reported having "enough space to walk around" (81\%) or "run around (10\%)". Only $6 \%$ reported that their physical space would limit their movement.

While Ma et al's work is promising in terms of reaching a representative sample and the environment in which participants take part in experiments, it suggests a difficulty in recruiting participants with high-end VR systems, which allow six-degrees of freedom (the ability to track user movement in real space) and leverage embodied controllers (e.g. Oculus Rift, HTC Vice). Only 18 (7\%) of eligible responses had a high-end VR system. A similar paucity of high-end VR equipment was found by Mottelson and Hornbæk [46], in which $1.4 \%$ of crowdworkers had access to these devices (compared to $4.5 \%$ for low-end devices, and $83.4 \%$ for Android smartphones). This problem is compounded if we consider Steed et al's finding that only $15 \%$ of participants provide completed sets of data [67].

An alternative approach to recruiting participants is to create experiments inside existing communities of XR users, such as inside the widely-used VR Chat software [60]. This allows researchers to enter into existing communities of active users, rather than attempt to establish their own. However, there are significant limitations for building experiments on platforms not designed for experimentation, such as programming limitations, the ability to communicate with outside services for data storage, and the absence of bespoke hardware interfaces.

\subsection{Remote data collection and experimentation}

2.3.1 Validity, benefits, drawbacks and differences. Using networks for remote data collection from human participants has been proven valid in some case studies [22,37]. In Gosling et al's comprehensive and well-cited study [22], internet-submitted samples were found to be diverse, generalise across presentation formats, were not adversely affected by non-serious or repeat respondents, and present results consistent with findings from in-lab methods. There is similar evidence for usability experiments, in which both the lab and remote tests captured similar information about the usability of websites [74].

That said, differences in results for lab and remote experiments are common $[10,64,70]$. The above website usability study also found that in-lab and remote experiments offered their own advantages and disadvantages in terms of the usability issues uncovered [74]. The factors that influence differences between in-lab and remote research are still being understood, but even beyond experiment design, there is evidence that even aspects such as the participant-perceived geographical distance between the participant and the data collection system influences outcomes [43].

Reips' [57] well-cited study outlined 18 advantages of remote experiments, including (l) easy access to a demographically and culturally diverse participant population, including participants from unique and previously inaccessible target populations; (2) bringing the experiment to the participant instead of the opposite; (3) high statistical power by enabling access to large samples; (4) the direct assessment of motivational confounding; and (5) cost savings of lab space, person-hours, equipment, and administration. He found seven disadvantages: (l) potential for multiple submissions, (2) lack of experimental control, (3) participant self-selection, (4) dropout, (5) technical variances, (6) limited interaction with participants and (7) technical limitations.

2.3.2 Supervised vs unsupervised. With the increasing availability of teleconferencing, it has become possible for researchers to be co"tele"present and supervise remote experiments through scheduling webcam experiment sessions. This presents a distinction from the unsupervised internet studies discussed above, and brings its own opportunities and limitations.

Literature broadly suggests that unsupervised experiments provide suitable quality data collection [26, 32,59]. A direct comparison between a supervised in-lab experiment and a large, unsupervised web-based experiment found that the benefits outweighed its potential costs [59]; while another found that a higher percentage of high-relevance responses came from unsupervised participants than supervised ones in a qualitative feedback setting [26]. There is also evidence that unsupervised participants react faster to tasks over the internet than those observed in the laboratory [32].

For longitudinal studies, research in healthcare has found no significant difference between task adherence rates between unsupervised and supervised groups [17]. However, one study noted that supervised studies had more effective outcomes [38].

2.3.3 Crowdworkers: Viable? Remote data collection was theorised to bring easy access to participants, including diverse participants and large samples [57]. Researchers have found that recruiting crowdworkers, people who work on tasks distributed to them over the internet, allowed them access to a large participant pool[49], with enough diversity to facilitate cross-cultural and international research [11]. Research has found that crowdworkers were significantly more diverse than typical American college samples and more diverse than other internet recruitment methods [11], at an affordable rate [49][11]. This has allowed researchers a faster theoryto-experiment cycle [42].

Results from crowdworker-informed studies have been shown to reproduce existing results from historical in-lab studies [49] [11] [66], while a direct comparison between experiment groups of crowdworkers, social media-recruited participants and on-campus recruitment, found almost indistinguishable results [13].

Some distinctions between crowdworkers and in-lab have been discovered, however. Comparative experiments between crowdworkers and in-person studies have suggested slightly higher participant rejection rates [66], while participants have been shown to report shorter narratives than other groups of college students (both online and in-person) and use proportionally more negative 
emotion terms than college students reporting verbally to an experimenter [24].

Distinctions also exist within crowdworker recruitment sources. A study of AMT, CrowdFlower (CF) and Prolific Academic (ProA) found differences in response rate, attention-check question results, data quality, honesty, diversity and how successfully effects were reproduced [50].

Data quality is a common concern regarding crowdworkers [21]. However, attention-check questions used to screen out inattentive respondents or to increase the attention of respondents have been shown to be effective in increasing the quality of data collected [3], as have participant reputation scores [51].

A growing concern regarding crowdworkers is non-naivete, in which participants having some previous knowledge of the study or similar studies that might bias them in the experiment. Many workers report having taken part in common research paradigms [48], and there are concerns that if researchers continue to depend on this resource, the problem may expand. As such, further efforts are needed by researchers to identify and prevent non-naive participants from participating in their studies [12].

\subsection{Summary}

It is clear that remote methods have been usefully deployed for non-XR research, and seemingly bring benefits such as easier participant recruitment, reduced recruitment cost and broadened diversity, without introducing major biases. However, there is still a paucity of research regarding the extent to which remote XR research can and has been used to leverage the unique benefits of both XR (environmental control, sensory illusions, data collection, replication) and remote (participation, practicality, cost-savings) methods, as well as the potential impact of their combined limitations. Therefore a survey of XR researcher experiences and beliefs regarding remote XR research could help us understand how these apply practically at the current time, and understand the key areas for future developments in this field.

\section{METHODOLOGY}

\subsection{Survey}

We surveyed current practice to outline the researcher-perceived benefits and drawbacks of lab-based and remote XR research. We used a 30-item qualitative questionnaire that enquired about participants' existing lab-based and remote research practices; thoughts on future lab-based and remote research; and potential benefits and drawbacks for each area. The survey was circulated through relevant mailing lists (visionlist@visionscience.com, BCS-HCI@ jiscmail.ac.uk, chi-announcements@listserv.acm.org), to members of groups thinking of or currently running remote studies, and to members of universities' virtual and augmented reality groups found via search engines.

Responses were thematically analysed using an inductive approach based upon Braun and Clarke's six phases of analysis [8]. The coding and theme generation process was conducted twice by independent researchers; themes were then reviewed collaboratively to create the final categorisations.

\subsection{Participants}

We received 46 responses to our survey from 36 different (predominantly academic) institutions. Most responses came from researchers based in Europe and North America, but responses also came from Asia. The majority of participants were either $\mathrm{PhD}$ students (18) or lecturers, readers or professors (11) at universities. Other roles were academic/scientific researcher (5), masters student (5), corporate researcher (4) and undergraduate student (2). A diverse set of ages responded to the survey: 18-24 (5), 25-34 (22), $35-44$ (11), 45+ (6), and gender skewed male (29) over female (16) or other (1).

\section{PARTICIPANT XR SETUP RESULTS}

Participants were more likely to have previously ran in-lab studies (37) than remote studies (14). Twenty-seven participants noted that, because of the Covid-19 pandemic, they have considered conducting remote XR experiments. In the next six months, more researchers were planning to run remote studies (24) than lab-based (22).

Participants predominantly categorised their research as VR-only (28) over AR-only (5). Ten participants considered their research as both VR and AR (and three did not provide an answer). This result

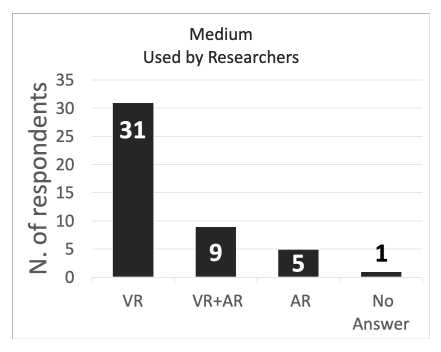

Figure 1: Type of XR medium explored by survey respondents.

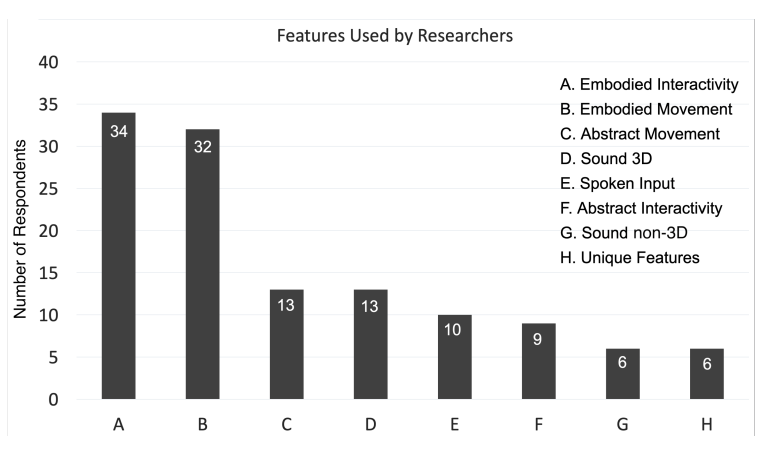

Figure 2: Features used by respondents in their user studies. (A) Embodied Interactivity: using embodied controller/camera-based movement. (B) Embodied Movement:using your body to move/"roomscale". (C) Abstract Movement: using a gamepad or keyboard and mouse to move. (D) Sound 3D: binaural acoustics. E) Spoken Input. (F) Abstract Interactivity: using a gamepad or keyboard and mouse to interact. (G) Sound non-3D: mono/stereo audio. (H) Unique features: e.g. haptics, hand tracking, scent. 
is illustrated in Fig. 1. In terms of research hardware, the majority of VR research leveraged virtual reality HMD-based systems with six degrees of freedom (32), that tracks participants' movements inside the room, over three degrees of freedom (15) or CAVE systems (1). Nineteen researchers made use of embodied or gesture controllers, where the position of handheld controllers are tracked in the real world and their position virtualised. For AR, HMDs were the predominant medium (13) over smartphones (9), with some researchers (5) using both.

An array of supplementary technologies and sensors were also reported by 13 respondents, including gaming joysticks, haptic actuators, a custom haptic glove, motion capture systems, e-textiles, eye-trackers, microphones, computer screens, Vive body trackers, brain-computer interfaces, EEG and electrocardiogram (ECG) devices, galvanic skin response sensors and hand-tracking cameras, as well as other spatial audio and hardware rigs.

The use of a variety of different off-the-shelf systems was also reported: Vive, Vive Pro, Vive Eye, Vive Index, Vive Pucks, Quest, Go, Rift, Rift S, DK2, Cardboard, Magic Leap One, Valve Knuckles, Hololens. Predominantly used devices are part of HTC Vive (25) and Oculus (23) family.

Respondents outlined numerous features of immersive hardware that they used in their research, visible in Fig. 2. The most prominent were embodiment aspects, including embodiment interactivity, in which a user's hand or body movements are reflected by a digital avatar (37) and embodiment movement (35), where participants can move in real space and that is recognised by the environment. Abstract movement (13), where a user controls an avatar via an abstracted interface (like a joystick) and abstract interactivity (8) were less popular. Spoken input was also used (10), as well as 3D sound (13) and non-3D sound (6). Scent was also noted (1) along with other unique features.

\section{THEMATIC ANALYSIS RESULTS}

In this section, we present and discuss the themes found in our survey study. The key points of each theme are summarised in a table at the start each subsection. Some of these points were found across multiple themes as they touch various aspects of user-based $\mathrm{XR}$ research.

\subsection{Theme: Study Sub-types}

Our analysis suggests that in-lab and remote studies can be additionally distinguished by whether the setting type is vital or preferred (summarised in Table 1). Broadly, in-lab (vital) studies require experimental aspects only feasible in-lab, such as bespoke hardware or unique data collection processes; in-lab (preferred) studies could take place outside of labs, but prefer the lab-setting based upon heightened concerns regarding the integrity of data collected and place a high value on a controlled setting. Remote (vital) studies are required when a user's natural environment is prioritised, such as explorations into behaviour in Social VR software; and remote (preferred) studies are used when cross-cultural feedback or a large number of participants are needed, or if the benefits offered by an in-lab setting are not required.

Beyond these, another sub-type emerged as an important consideration for user studies: supervised or unsupervised. While less of an
Table 1: Summary of XR Study Sub-types

\begin{tabular}{ll}
\hline Method & Summary \\
\hline In-lab (vital) & $\begin{array}{l}\text { Experiment requires features only feasible } \\
\text { in-lab, e.g. bespoke hardware, unique data } \\
\text { collection } \\
\text { Concerns about integrity of data collected } \\
\text { In-lab (preferred) }\end{array}$ \\
$\begin{array}{l}\text { remotely, high value on controlled setting } \\
\text { Remote (vital) }\end{array}$ & $\begin{array}{l}\text { important (e.g. Social VR, naturally experi- } \\
\text { enced at home and online) } \\
\text { Priority to get cross-cultural feedback or } \\
\text { reach large number of participant; lab pro- } \\
\text { vides limited benefits }\end{array}$ \\
\hline
\end{tabular}

important distinction for in-lab studies (which are almost entirely supervised), participant responses considered both unsupervised "encapsulated" studies, in which explanations, data collection and the study itself exist within the software or download process, and supervised studies, in which researchers schedule time with the remote participant to organise, run and/or monitor the study. These distinctions will be discussed in more detail throughout the analysis below, as the sub-types have a distinct impact on many of the feasibility issues relating to remote studies.

\subsection{Theme: Study Participants}

5.2.1 Recruitment scope. Twenty-nine respondents stated the wellknown challenge of recruiting a satisfactory number of participants for lab-based studies. Issues were reported both with the scale of available participants, and the problem of convenience sampling and WEIRD - Western, educated, industrialized, rich and democratic societies - participants[25].

Participant recruitment was mentioned by 27 respondents as the area in which remote user studies could prove advantageous over labs. Remote studies could potentially provide easier recruitment (in terms of user friction: accessing to lab, arriving at the correct time), as well as removing geographic restrictions to the participant pool.

Removing the geographic restrictions also simplifies researchers' access to cross-cultural investigations (R23, R43). While cross-cultural lab-based research would require well-developed local recruitment networks, or partnerships with labs in target locations, remote user studies, and more specifically, systems built deliberately for remote studies, introduce cross-cultural scope at no additional overhead.

There are, however, common concerns over the limitations to these benefits due to the relatively small market size of XR technologies. For AR, this is not a strong limitation for smartphones-based explorations, but the penetration of HMD AR and VR technology is currently limited, and it is possible that those who currently have access to these technologies will not be representative of the wider populations. Questions remain over who the AR/VR HMD owners are, if they exhibit notable differences from the general population, and if those differences are more impactful than those presented by existing convenience sampling. 
Table 2: Study Participants Key Points

\begin{tabular}{llll}
\hline Key Point & Issue & Lab & Remote \\
\hline $\begin{array}{l}\text { Recruitment Scope } \\
\text { Recruitment Scope }\end{array}$ & $\begin{array}{l}\text { Sample size } \\
\text { Sample balance }\end{array}$ & $\begin{array}{l}\text { Usually smaller numbers } \\
\text { Might be easier to ensure balance }\end{array}$ & $\begin{array}{l}\text { Potential for larger number } \\
\text { How to ensure balance? (e.g. who mostly owns XR } \\
\text { equipment?) }\end{array}$ \\
Efficiency & Time & $\begin{array}{l}\text { Requires setup time and organise par- } \\
\text { ticipants }\end{array}$ & $\begin{array}{l}\text { Potential less time especially if encapsulated and } \\
\text { unsupervised }\end{array}$ \\
$\begin{array}{l}\text { Precursor Require- } \\
\text { ments }\end{array}$ & Requisites & $\begin{array}{l}\text { Pre-test and linguistic/culture compre- } \\
\text { hension conditions are ensured }\end{array}$ & Not clear how to verify conditions in remote studies \\
\hline
\end{tabular}

Despite the belief that designing for remote participants will increase participant numbers, and therefore the power of studies, it seems unclear how researchers will reach HMD-owning audiences. Thirty respondents who have, or plan to, run remote XR studies have concerns about the infrastructure for recruiting participants remotely. Unlike other remote studies, the requirement for participants to own or have access to XR hardware greatly reduces the pool (around 5 million XR HMDs were sold in 2020 [73]). A major outstanding question is how researchers can access these potential participants, although some platforms for recruiting XR participants have emerged in the past few months such as XRDRN.org.

Nine respondents noted that remote XR experiments may encourage participation from previously under-represented groups, including introverts and those who cannot or do not wish to travel into labs to take part (e.g. people who struggle to leave their homes due to physical or mental health issues).

However, respondents with research-specific requirements also raised concerns that recruitment of specific subsets of participants could be more difficult remotely. For example, when recruiting for a medical study of those with age-related mobility issues, it is unlikely that there will be a large cohort with their own XR hardware.

5.2.2 Theme: Efficiency. Twenty-five respondents noted the potential for remote studies to take up less time, particularly if remote studies are encapsulated and unsupervised. They stated that this removes scheduling concerns for both the researcher and the participant, and allows experiments to occur concurrently, reducing the total researcher time needed or increasing the scale of experiment. However, there are concerns this benefit could be offset by increased dropouts for longitudinal studies, due to a less "close" relationship between research and participant (R17, R25).

5.2.3 Participant precursor requirements. One respondent noted they needed to run physiological precursor tests (i.e. visual acuity and stereo vision) that have no remote equivalent. Transitioning to remote research has meant this criteria must now be self-reported. Similarly, experiments have general expectations of linguistic and cultural comprehension, and opening research to a global scale might introduce distinctions from typically explored population. One respondent cautioned that further steps should be taken to ensure participants are able to engage at the intended level, as in-lab these could be filtered out by researcher intuition.

\subsection{Data Collection}

The overwhelming drawback of remote XR research, as reported by the majority respondents, was that of data collection. Excluding changes to participant recruitment, as mentioned above, the issues can broadly be categorised as: (1) bespoke hardware challenges, (2) monitoring/sensing challenges, and (3) data transmission and storage.

The use of bespoke hardware in any type of remote user study is a well-known issue, predominantly regarding the difficulty of managing and shipping bespoke technology to participants and ensuring it works in their test environments. In the context of XR technologies, 13 respondents voiced concerns about the complicated and temperamental system issues that could arise, particularly surrounding the already strenuous demands of PC-based VR on consumer-level XR hardware, without additional overheads (e.g. recording multiple cameras).

Four respondents felt it was unreasonable to ask remote participants to prepare multiple data-collection methods that may be typical in lab-studies, such as video recording and motion tracking. There were also concerns regarding the loss of informal, ad-hoc data collection (e.g. facial expressions, body language, casual conversations).

Finally, concerns were also raised regarding the efforts required to encapsulate all data capture into the XR experience, the effects this might have on data collection (for example, a recent study highlighted a difference on the variability of presence when participants recorded it from inside the VR experience versus outside [62]), the reliability of transferring large amounts of data from participants, and how sensitive information (especially in the context of medical XR interventions) can securely be transferred and stored. This areas perhaps presents the biggest area for innovation for remote XR research, as it is reasonable to assume the academic community could create efficient, easy-to-use toolkits for remote data collection in XR environments which integrate to ethics-compliant data archives.

Many data collection methods were deemed infeasible for remote experimentation: EEG, ECG, eye/hand tracking, GSR, as well as body language and facial expressions. Five researchers noted adaptions they had been working on to overcome these, including using HMD orientation to replace eye tracking, and using built-in HMD microphones to record breaths instead of ECG monitoring to determine exertion, or using the HMD controllers to perform hand tracking. 
Table 3: Data Collection Key Points

\begin{tabular}{|c|c|c|}
\hline Key Point & $\mathrm{Lab}$ & Remote \\
\hline Hardware & Access custom and/or reliable hardware & $\begin{array}{l}\text { Limited access to devices (e.g. EEG, ECG, computational } \\
\text { power, etc.) }\end{array}$ \\
\hline Data & $\begin{array}{l}\text { Collection can be supervised, more detailed, real-time, } \\
\text { more space for qualitative }\end{array}$ & $\begin{array}{l}\text { Mostly unsupervised (less control), human expressions } \\
\text { (e.g. facial) are generally lost, qualitative feedback is } \\
\text { harder to collect }\end{array}$ \\
\hline Behaviour & Likely more serious, richer (qualitative) data & Lack of detailed feedback, potentially less honest \\
\hline
\end{tabular}

Respondents also noted some behavioural concerns and changes for remote, unsupervised participants. These included a lack of participation in qualitative feedback (6 respondents); for one researcher (R20), participants were "encouraged to provide feedback but few took the initiative." Another researcher (R31) stated "Debriefing is such a good space to collect unstructured interview data. Users relax after the questionnaire/debriefing ... produc[ing] a ... meta-narrative where participants consider your questions and their experiences together". The lack of supervision raised concerns regarding whether participants were being "truthful" in their responses, with one researcher (R41) stating that participants attempted to "game" their study in order to claim the participation compensation. However, others stated that unsupervised studies could reduce research bias arising from their perception of the participants' appearance and mannerisms.

\subsection{Theme: Experiment Processes}

5.4.1 Process \& Guidance. Many respondents were concerned that unsupervised participants may conduct the experiments incorrectly, or have incorrect assumptions, or misunderstand processes or target actions. Twenty-four respondents felt that guidance would be better provided (introduction, explanations, etc) in a lab setting that also allows ad-hoc guidance and real-time corrections.

There were also concerns over the mental state of participants: remote participants "may not take it seriously" or not focus (lack of motivation and engagement) or approach the study with a specific mood unknown to the researcher (R19, R30). Contrasting opinions suggested that participants may feel that the in-lab experience is "overly formal and uncomfortable" (R32).

Some respondents stated that remote experiments risk losing the "rapport" between researcher and participant, which might negatively influence the way a participant performs a remote study. However, one respondent stated that the transition to remote experimentation allowed them different, deeper, on-going connection with their participants. Their research was for a VR machine learning tool, and they found that moving away from in-person experimentation and to a remote workshop process encouraged the up-take of longitudinal community-building tools. The chosen communication method between researcher and user - Discord servers - became a place for unsupervised interaction between participants, and led to an on-going engagement with the research (R33). However it should be considered that any "rapport" between participant and researcher might introduce bias.
5.4.2 Environment. Concern was raised around participants' environments, and their potential varying unsuitability for remote experimentation, compared with controlled laboratory settings. For example, one respondent (R20) stated: "one user reported walking into their whiteboard multiple times, causing low presence scores." The concern is particularly strong for unsupervised remote experiments, as distractions could enter into the experiment and affect data without the researcher being aware.

This concern was not universal, however. Four respondents noted that their laboratories space was far from distraction free, and even suggested that a remote space could prove freer of interruptions than the space available to them in their research setting; while others stated that researchers should be mindful that the laboratory itself is an artificial space, far more so than where people will typically use their VR setups - in their homes. Five respondents highlighted how XR research could benefit from being deployed in "the participants' own environment".

The immediate environment of the user was also raised as a concern for VR experiment design: the choice of being able to move freely in an open space in a laboratory against a more adaptive solution for the unknown variables of participants' home environments.

Respondents noted that supporting the different VR and AR setups to access a larger remote audience would also prove more labour-intensive, and would introduce more variables compared with the continuity of the tech stack available in-lab. With remote experiments, and more so for encapsulated unsupervised ones, 10 respondents believe there will be more time spent in developing the system.

5.4.3 Hardware and software. A concern regarding remote experiments, particularly unsupervised, is that calibration processes are harder to verify (R30). This could either cause participants to unknowingly have faulty experiences, and therefore report faulty data; or it will increase time taken to verify user experiences are correct. Unknown errors can effect data integrity or participant behaviour. Respondents noted that this type of remote error are often much more difficult and labour-intensive to fix compared with in-lab. This issue is compounded by individual computer systems introducing other confounding factors (for both bug-fixing and data collection) such as frame-rates, graphic fidelity, tracking quality and even resolution can vary dramatically.

Five respondents reflected that overcoming these issues could lead to more robust research plans, as well as better development 
Table 4: Experiment Process Key Points

\begin{tabular}{|c|c|c|c|}
\hline Key Point & Issue & $\mathrm{Lab}$ & Remote \\
\hline Process \& Guidance & Control & Full control over setup and participants & No control and guidance over participants \\
\hline Process \& Guidance & Participants & $\begin{array}{l}\text { Rapport with researcher, welcoming, more } \\
\text { serious, attentive }\end{array}$ & Different attitude, potential cheating \\
\hline Environment & Setting & $\begin{array}{l}\text { Can be distracting (e.g. outside noise) but } \\
\text { generally more controlled }\end{array}$ & $\begin{array}{l}\text { Might be distracting or overwhelming but } \\
\text { likely more realistic/natural for participants }\end{array}$ \\
\hline Hardware \& software & Hardware & $\begin{array}{l}\text { Access to custom devices, normal calibra- } \\
\text { tion process }\end{array}$ & $\begin{array}{l}\text { No calibration (by researcher), potential for } \\
\text { unknown errors, no custom tools }\end{array}$ \\
\hline Hardware \& software & Software & $\begin{array}{l}\text { Allows for Wizard of } \mathrm{Oz} \text {, adjust setting in } \\
\text { real time }\end{array}$ & $\begin{array}{l}\text { Issues harder to spot and influence results } \\
\text { longer development time }\end{array}$ \\
\hline Research questions & Topics & $\begin{array}{l}\text { Unchanged, if we go back to normal re- } \\
\text { search conditions }\end{array}$ & $\begin{array}{l}\text { Remote setup might influence research } \\
\text { questions and topics }\end{array}$ \\
\hline Cost & Expenditures & $\begin{array}{l}\text { More time consuming, more expensive to } \\
\text { run }\end{array}$ & $\begin{array}{l}\text { Potentially cheaper but potentially more } \\
\text { work for implementation }\end{array}$ \\
\hline
\end{tabular}

and end-product software to overcome problems listed. This encapsulation could also lead to easier opportunities for reproducability, as well as the ability for researchers to share working versions of the experiment with other researchers, instead of just the results. It could also help with the versioning of experiments, allowing researchers to build new research on-top of previous experiment software.

Four respondents were aware these advantages are coupled with longer development times. The increased remote development requirements could also be limiting for researchers who face constrained development resources, particularly those outside of computer science departments. This is compounded by the fact that the infrastructure for recruiting remote XR participants, data capture, data storage and bug fixing is not particularly developed. Once these are established, however, respondents felt these might make for a higher overall data quality compared with the current laboratorybased status quo, due to more time spent creating automated recording processes, and not relying on researcher judgement. There are also arguments that the additional development time is offset by the potential increase in participants and, if unsupervised, the reduction in experiment supervision requirements.

Six respondents that use specific hardware in their research, noted that it was currently difficult to measure physiological information in a reliable way, and included hand tracking in this. However, we are aware that some consumer VR hardware (Oculus Quest) allows hand-tracking, and so there is an additional question of whether researchers are being fully supported in knowing what technologies are available to them.

To alleviate issues with reaching participants, two respondents wrote about potentially sending equipment to participants. The limitations of this were noted as hardware having gone missing (which had happened, R35), and participants being unable to use equipment on their own (which had not happened yet).

5.4.4 Research questions. Five respondents noted that their research questions changed or could change depending on whether they were aiming for a laboratory or remote settings. For example, one respondent (R31) suggested that "instead of the relationship

\section{Table 5: Health and Safety Key Points}

\begin{tabular}{ll}
\hline Key Point & Summary \\
\hline Protocols & $\begin{array}{l}\text { Missing standard protocols (to work safely } \\
\text { with participants in-lab) }\end{array}$ \\
Equipment & $\begin{array}{l}\text { Sanitizing of in-lab equipment and spaces } \\
\text { Concerns for remote participants (e.g. acci- } \\
\text { Remote }\end{array}$ \\
dents during a user study) \\
Real-Time Aid & $\begin{array}{l}\text { Not available for remote participants (e.g. } \\
\text { motion sickness) }\end{array}$ \\
\hline
\end{tabular}

of the physical body to virtual space, I'd just assess the actions in virtual space". Others explored the potentiality of having access to many different system setups, for example, now being able to easily ask questions like "are there any systematic differences in cybersickness incidence across different HMDs?". (R39)

Nine respondents speculated that remote research has potential for increasing longitudinal engagement, due to lower barriers to entry for researcher (room booking, time) and participant (no commute), and that rare or geographically based phenomena could be cheaply studied using remote research; as providing those communities access to VR may be cheaper than relocating a researcher to them.

5.4.5 Costs. Eight respondents noted the potential of remote experimentation for reducing some of the cost overheads for running experiments. Laboratories have important costs that are higher than remote studies: lab maintenance, hardware maintenance, staff maintenance. Without these, costs per participant are lower (and for unsupervised studies, almost nil). As experiment space availability was also noted as a concern for laboratory-based experiments, this seems a potentially under-explored area of benefit, provided remote participant recruitment is adequate. 


\subsection{Theme: Health \& Safety}

The leading benefit given for remote user studies was that of health and safety, citing shared HMDs and controllers as a potential vector for Covid-19 transmission, as well as more general issues such as air quality in enclosed lab spaces. Concerns were raised for both viral transmission between participants, and between participant and the researcher. This concern has also increased administration overheads, with 6 respondents stating it could be more time consuming to prepare the lab and organise the studies or using new contract-tracing methods for lab users.

However, respondents also raised concerns about additional safety implications for remote participants. The controlled lab environment is setup to run the study, whereas remote participants are using a general-purpose space. One AR researcher who conducts research that requires participants to move quickly outside in fields noted his study could be considered "incredibly unsafe" if unsupervised or run in an inappropriate location. Additionally, for health and mental health studies, in-lab allows for researcher to provide support, especially with distressing materials. Finally, VR environment design has a direct impact on the level of simulator sickness invoked in participants. There were questions about the responsibility of researchers to be present to aid participants who could be made to feel unwell from a system they build.

\subsection{Theme: Ethics}

Three ethics concerns were reported by respondents: encouraging risky behaviours, responsibility for actions in XR and data privacy. An example of this might be the ethical implications of paying participants, and therefore incentivising them, to take part in what could be considered a high-risk behaviour: entering an enclosed space with a stranger and wearing a VR HMD.

Respondent (R30) raised the question of liability for participants who are injured in their homes while taking part in an XR research project. The embodied nature of XR interventions - and most respondents used this embodiment in their studies - could put participants at a greater risk of harming themselves than with other mediums.

Finally, while cross-cultural recruitment was seen as a potential boom for remote research, questions were raised about ethics and data storage and protection rules when participants are distributed across different countries, each with different data storage laws and guidelines. Although not limited to XR, due to the limited number of VR users, and the disproportionate distribution of their sales, it seems the majority of remote VR participants will originate from North America, and ethics clarification from non-US-based universities are needed.

\section{COVID-19 IMPLICATIONS}

While Covid-19 has impacted most studies around the world, the dependence on shared hardware for XR research, especially HMDs, has led to many implications reported by our respondents. These concerns are particularly related to Covid-19, and therefore be reduced as the pandemic is resolved. However, as it is currently unclear when the pandemic will end, we felt it was useful to discuss them in a dedicated section.

Most respondents noted that Covid-19 had caused a suspension of studies and that they were unclear how long the suspension
Table 6: Covid-19 Implications

\begin{tabular}{ll}
\hline Key Point & Summary \\
\hline Suspensions & No user studies at the moment \\
Facilities & Sanitizing of equipment and spaces \\
Recruitment & $\begin{array}{l}\text { Harder/impossible to recruit in-lab partici- } \\
\text { pants }\end{array}$ \\
Exclusion & Bias and high risk participants \\
\hline
\end{tabular}

would last for, resulting in an overall drop in the number of studies being conducted, with 30 respondents stating it will change the research they conduct (e.g. moving to online surveys). The continuation of lab studies was eventually expected, but with added sanitizing steps. However for many, it was unclear what steps they should take in order to make XR equipment sharing safe. These concerns extended beyond the XR hardware to general facility suitability, including room airflow and official protocols which may vary for each country and/or institution.

Five respondents also had concerns about participants. There were worries that lab-based recruitment would be slow to recover, as participants may be put off taking part in experiments because of the potential virtual transfer vectors. Similarly, respondents were concerned about being responsible for participants, and putting them in a position in which their is a chance they could be exposed to the virus.

There was also concerns around Covid-19 and exclusion, as researchers who are at high risk of Covid-19 or those who are in close contact with high risk populations, would now have to selfexclude from lab-based studies. This might introduce a participant selection bias towards those willing to attend a small room and sharing equipment,

It should be noted that not all labs are facing the same problems - some of our respondents had continued lab-based experimentation during this period, with Covid-19 measures ensuring that participants wore face masks during studies. This was considered a drawback as combined with an HMD, it covered the participant's entire face and was cumbersome. These measures are also known not to be $100 \%$ protective.

\section{DISCUSSION}

In the previous section we presented the results as themes we found in our analysis. Some of these presented common characteristics and some issues were reported in multiple themes. We now summarise the results, highlight the key points and suggest important questions for future research.

\subsection{Recruitment and participants}

As with non-XR experiments, researchers are interested in the potential benefits of remote research for increasing the amount, diversity and segmentation of participants compared with in-lab studies. However, with many respondents reporting that it has been difficult to recruit XR participants, it seems there is a gap between potential and practice. The unanswered question is how to build a pool of participants that is large and diverse enough to accommodate various XR research questions and topics, given 
that there are few high-end HMDs circulating in the crowdworker community [40][46]. So far, we have found three potential solutions for participant recruitment, although each requires further study:

(1) Establish a dedicated XR crowdworker community. However, concerns of non-naivety[48], which are already levied at the much larger non-XR crowdworker participant pools, would surely be increased. We would also have to understand if the early version of this community would be WEIRD[25] and non-representative, especially given the cost barrier to entry for HMDs.

(2) Leverage existing consumer XR communities on the internet, such as the large discussion forums on Reddit. These should increase in size further as they shift from early-adopter to general consumer communities. However, these communities may also have issues with representation.

(3) Establish hardware-lending schemes to enable access to a broader base of participants [68]. However, the cost of entry and risk of these schemes may make them untenable for smaller XR research communities.

It is also not clear, beyond HMD penetration, what the additional obstacles are that XR poses for online recruitment. Technical challenges (e.g. XR applications needing to run on various devices, on different computers, requiring additional setup beyond simple software installation) and unintuitive experiment procedures (e.g download $\mathrm{X}$, do an online survey at $\mathrm{Y}$, run $\mathrm{X}$, record $\mathrm{Z}$ ) for participants are notable distinct issues for remote $\mathrm{XR}$ research. It is also unclear if the use of XR technology has an impact on what motivates participants to take part in remote studies, an area of study that has many theoretical approaches even in the non-XR area[33].

\subsection{Data collection}

Respondents feel that many types of physiological data collection are not feasible with either XR or non-XR remote research. For remote XR research, there are unique concerns over video and qualitative data collection as using XR technologies can make it (technically) difficult to reliably video or record the activity, as well as moving participants' loci of attention away from the camera or obscuring it behind an HMD. However, the hardware involved in creating XR experiences provides a variety of methods to gather data, such as body position, head nodding, breath-monitoring, hand tracking, HMD angle instead of eye tracking. These can be used to explore research topics that are often monitored via other types of physiological, video or qualitative data, such as attention, motivation, engagement, enjoyment, exertion or focus of attention. It would be useful for XR researchers to build an understanding of what the technologies that are built into XR hardware can tell us about participant experiences, so as to allow us to know the data collection affordances and opportunities of XR hardware.

That said, the infrastructure for collecting and storing this (mass) of XR data remotely is currently not fully implemented, and we are not aware of any end-to-end standardised framework. However, work is being done to simplify the data collection step for XR experiments build in Unity [9]. There are also opportunities to further develop web-based XR technologies that could send and store data on remote servers easily. There are also ethical concerns, as respondents were unclear on guidance regarding data collection from participants located in other nations, particularly when they should be paid. This includes how the data is collected, where it should be stored, and how can be manipulated.

\subsection{Health, safety and Covid-19}

At the time of writing, many laboratories are considered unsafe for running user studies. Although some respondents reported being able to work in-lab, the limitations mean it is not currently feasible to run user studies under normal conditions. The main concern for the near future is the lack of standardised protocols to ensure safety of researchers and participants while running user studies and the issue with the ethics protocols of the research institutions. For XR research, it is unclear how to adequately sanitize equipment and tools, as well as how to maintain physical distancing. There are also concerns about the comfort of participants if they are required to wear masks alongside HMDs. Finally, respondents reported concerns about a potential long-term fall in user motivation to take part in such experiments, when HMDs are a notable infection vector. There are distinctly different safety and ethics concerns around remote XR experiments, including the research responsibility for not harming participants (e.g. ensuring environments are safe for the movements, and not inducing simulator sickness), which, while also true of in-lab experiments, are considered a greater challenge when a participant is not co-located with the researcher.

\subsection{Mediated impact}

Respondents reported framing their research questions and experiments differently depending on the target experiment setting. The strongest transition was that of an in-lab study of participants using an AR HMD (Hololens), which changed to a remote study that had participants watch a pre-recorded video of someone using the AR HMD. It seems these kinds of transitions will continue to be necessary depending on how esoteric the hardware is, with fewer concerns for AR smartphone investigations.

A concern for respondents was that remote settings introduce additional uncontrolled variables that need to be considered by researchers, such as potential unknown distractions, trust in participants and their motivation, and issues with remote environmental spaces. However, previous research shows that most HMD-wearing remote participants engage in space well-known to them (the home) and predominantly when they are alone [40], which could alleviate some of the environmental space and distraction concerns. Further research into how a home environment could impact XR studies is needed, and the creation of well-defined protocols to alleviate uncontrolled influences remote XR results. Beyond this, we also need to understand any impact that remote experiments may have on results compared with in-lab experiences, especially if we are to be able to reliably contrast lab and remote research. Previous research for non-XR experiments suggest that distinctions between lab and remote settings exist [10][64] [70], but it has been theorised that the impact might be less for XR experiments, as you "take the experimental environment with you" [7].

\section{5 "Encapsulated" experiments: the ideal?}

Respondents stated that creating remote XR experiments might encourage better software development and experimental processes. 
If experiments are able to be deployed as all-in-one experience and data collection bundles that can run unsupervised, the time-saving implications for researchers (and participants) are huge, especially when paired with the potential increase in participants. This type of "encapsulated experiment" can also improve replication and transparency, as theorised by Blascovich [7], and allow for versioning of experiments, in which researchers can build on perfect replicas of other's experimental environments and processes. Finally, due to the similar nature of XR hardware, data logging techniques could easily be shared between system designers or standardised; something we have seen with the creation of the Unity Experiment Framework [9].

However, there are some limitations to this approach. It is likely it will require additional development time from the researchers, especially as a comprehensive experiment framework is established. In addition, there are data collection limitations for remote XR studies, as discussed in previous sections. It is also interesting to consider how encapsulation might work for AR investigations, as the environment will only partially be controlled by the designer.

We believe that the potential for remote XR experiments lies in understanding the data collection affordances of the hardware; collectively building frameworks to ease the collection of this data; and to design research questions that maximise their use; all inside encapsulated experiences. This might be a mindset shift for researchers, who according to our survey, are predominantly laborientated.

\section{LIMITATIONS}

Our goal with this research was to provide an overall insight into the XR researcher community. However, this approach means that insights from sub-communities may not have been found. For example, we had no responses from researchers involved in topics such as vulnerable populations. Further investigation into sub-communities is needed to uncover potential insights for those areas.

\section{CONCLUSION AND RECOMMENDATIONS}

It is clear from our survey that respondents believe that remote XR research has the potential to be a useful research approach. However, it currently suffers from numerous limitations regarding data collection, system development and a lack of clarity around participant recruitment. Analysis of our survey results and literature around remote and remote XR research suggest that, to better understand the boundaries of remote XR experimentation, researchers need answers to the following questions:

(1) Who are the potential remote XR participants, and are they representative?

(2) How can we access a large pool of remote XR participants?

(3) To what extent do remote XR studies affect results compared with in-lab?

(4) What are the built-in XR data collection affordances of XR hardware, and what can they help us study?

(5) How can we lower the barriers to creating encapsulated experiment software, to maximise the potential of remote $\mathrm{XR}$ research?
We believe there is an opportunity to reconceptualise approaches to $\mathrm{XR}$ and remote research. XR experiments, as it stands, are predominantly used to study a participant's experience with an XR system, in an artificial but controlled setting (laboratory) using external data collection methods (surveys, cameras, etc.). However, if we consider XR devices primarily as data-collection hardware with set properties, we can work backwards to understand what research questions are suitable with the existing data collection afforded by $\mathrm{XR}$ hardware. Additionally, we also believe that there is potential to reconceptualise, for suitable applications, the home as a natural research location and move away from the laboratory as the default location for user studies. This is a potentially unique opportunity for XR compared with non-XR studies as, for many investigations, the XR experiment takes the environment with it.

\section{ACKNOWLEDGMENTS}

This work is supported by the EPSRC and AHRC Centre for Doctoral Training in Media and Arts Technology (EP/L01632X/1).

\section{REFERENCES}

[1] Judith Amores, Robert Richer, Nan Zhao, Pattie Maes, and Bjoern M Eskofier. 2018. Promoting relaxation using virtual reality, olfactory interfaces and wearable EEG. In 2018 IEEE 15th international conference on wearable and implantable body sensor networks (BSN). IEEE, IEEE, Las Vegas, 98-101.

[2] Elliot Aronson. 1969. The theory of cognitive dissonance: A current perspective. Advances in experimental social psychology 4, 1 (1969), 34

[3] Frederik Aust, Birk Diedenhofen, Sebastian Ullrich, and Jochen Musch. 2013. Seriousness checks are useful to improve data validity in online research. Behavior research methods 45, 2 (2013), 527-535.

[4] Jakki Bailey, Jeremy N Bailenson, Andrea Stevenson Won, June Flora, and K Carrie Armel. 2012. Presence and memory: immersive virtual reality effects on cued recall. In Proceedings of the International Society for Presence Research Annual Conference. Citeseer, International Society for Presence Research Annual Conference, Philadelphia, Pennsylvania, USA, 24-26.

[5] Domna Banakou, Parasuram D Hanumanthu, and Mel Slater. 2016. Virtual embodiment of white people in a black virtual body leads to a sustained reduction in their implicit racial bias. Frontiers in human neuroscience 10 (2016), 601.

[6] Jim Blascovich. 2002. Social influence within immersive virtual environments. In The social life of avatars. Springer, California, USA, 127-145.

[7] Jim Blascovich, Jack Loomis, Andrew C Beall, Kimberly R Swinth, Crystal L Hoyt, and Jeremy $\mathrm{N}$ Bailenson. 2002. Immersive virtual environment technology as a methodological tool for social psychology. Psychological inquiry 13, 2 (2002), 103-124.

[8] Virginia Braun and Victoria Clarke. 2006. Using thematic analysis in psychology. Qualitative research in psychology 3, 2 (2006), 77-101.

[9] Jack Brookes, Matthew Warburton, Mshari Alghadier, Mark Mon-Williams, and Faisal Mushtaq. 2019. Studying human behavior with virtual reality: The Unity Experiment Framework. Behavior research methods 1, 52 (2019), 1-9.

[10] Tom Buchanan. 2000. Potential of the Internet for personality research. In Psychological experiments on the Internet. Elsevier, Wir 8AL United Kingdom, 121-140.

[11] Michael Buhrmester, Tracy Kwang, and Samuel D Gosling. 2016. Amazon's Mechanical Turk: A new source of inexpensive, yet high-quality data? Perspectives on Psychological Science 1, 6 (2016), 3-5.

[12] Michael D Buhrmester, Sanaz Talaifar, and Samuel D Gosling. 2018. An evaluation of Amazon's Mechanical Turk, its rapid rise, and its effective use. Perspectives on Psychological Science 13, 2 (2018), 149-154.

[13] Krista Casler, Lydia Bickel, and Elizabeth Hackett. 2013. Separate but equal? A comparison of participants and data gathered via Amazon's MTurk, social media, and face-to-face behavioral testing. Computers in human behavior 29, 6 (2013), 2156-2160.

[14] Woong Choi, Liang Li, Satoru Satoh, and Kozaburo Hachimura. 2016. Multisensory integration in the virtual hand illusion with active movement. BioMed research international 2016 (2016), 9.

[15] Richard E Clark and Terrance G Craig. 1992. Research and theory on multimedia learning effects. In Interactive multimedia learning environments. Springer, University of Southern California USA, 19-30.

[16] Lauri Connelly, Yicheng Jia, Maria L Toro, Mary Ellen Stoykov, Robert V Kenyon, and Derek G Kamper. 2010. A pneumatic glove and immersive virtual reality 
environment for hand rehabilitative training after stroke. IEEE Transactions on Neural Systems and Rehabilitation Engineering 18, 5 (2010), 551-559.

[17] S. A. Creasy, R. J. Rogers, K. K. Davis, B. B. Gibbs, E. E. Kershaw, and J. M. Jakicic 2017. Effects of supervised and unsupervised physical activity programmes for weight loss. Obesity Science \& Practice 3, 2 (2017), 143-152. https://doi.org/10 1002/osp4.107 arXiv:https://onlinelibrary.wiley.com/doi/pdf/10.1002/osp4.107

[18] Darragh Egan, Sean Brennan, John Barrett, Yuansong Qiao, Christian Timmerer, and Niall Murray. 2016. An evaluation of Heart Rate and ElectroDermal Activity as an objective QoE evaluation method for immersive virtual reality environments. In 2016 Eighth International Conference on Quality of Multimedia Experience (QoMEX). IEEE, IEEE, Lisbon, Portugal, 1-6.

[19] Donald P Ely and Barbara B Minor. 1994. Educational Media and Technology Yearbook, 1994. Volume 20. ERIC, 6633, Englewood, CO.

[20] Daniel Freeman, Polly Haselton, Jason Freeman, Bernhard Spanlang, Sameer Kishore, Emily Albery, Megan Denne, Poppy Brown, Mel Slater, and Alecia Nickless. 2018. Automated psychological therapy using immersive virtual reality for treatment of fear of heights: a single-blind, parallel-group, randomised controlled trial. The Lancet Psychiatry 5, 8 (2018), 625-632.

[21] Joseph K Goodman, Cynthia E Cryder, and Amar Cheema. 2013. Data collection in a flat world: The strengths and weaknesses of Mechanical Turk samples. fournal of Behavioral Decision Making 26, 3 (2013), 213-224.

[22] Samuel D Gosling, Simine Vazire, Sanjay Srivastava, and Oliver P John. 2004 Should we trust web-based studies? A comparative analysis of six preconceptions about internet questionnaires. American psychologist 59, 2 (2004), 93

[23] Diane Gromala, Xin Tong, Amber Choo, Mehdi Karamnejad, and Chris D Shaw. 2015. The virtual meditative walk: virtual reality therapy for chronic pain management. In Proceedings of the 33rd Annual ACM Conference on Human Factors in Computing Systems. Association for Computing Machinery, New York, NY, USA 521-524.

[24] Azriel Grysman. 2015. Collecting narrative data on Amazon's Mechanical Turk. Applied Cognitive Psychology 29, 4 (2015), 573-583.

[25] Joseph Henrich, Steven J Heine, and Ara Norenzayan. 2010. Most people are not WEIRD. Nature 466, 7302 (2010), 29-29.

[26] M. Hertzum, Pia Borlund, and K. B. Kristoffersen. 2015. What Do Thinking Aloud Participants Say? A Comparison of Moderated and Unmoderated Usability Sessions. International fournal of Human-Computer Interaction 31 (2015), 557 570 .

[27] Hunter G Hoffman, Azucena Garcia-Palacios, Veronica Kapa, Jennifer Beecher and Sam R Sharar. 2003. Immersive virtual reality for reducing experimental ischemic pain. International fournal of Human-Computer Interaction 15, 3 (2003), 469-486.

[28] Crystal L Hoyt, Jim Blascovich, and Kimberly R Swinth. 2003. Social inhibition in immersive virtual environments. Presence: Teleoperators \& Virtual Environments 12, 2 (2003), 183-195.

[29] Bernd Huber and Krzysztof Z Gajos. 2020. Conducting online virtual environment experiments with uncompensated, unsupervised samples. Plos one 15, 1 (2020) $\mathrm{e} 0227629$.

[30] Naomi Josman, Eli Somer, Ayelet Reisberg, Patrice L Weiss, Azucena GarciaPalacios, and Hunter Hoffman. 2006. BusWorld: designing a virtual environment for post-traumatic stress disorder in Israel: a protocol. Cyberpsychology \& Behav ior 9, 2 (2006), 241-244.

[31] Roland Kehl and Luc Van Gool. 2004. Real-time pointing gesture recognition for an immersive environment. In Sixth IEEE International Conference on Automatic Face and Gesture Recognition, 2004. Proceedings. IEEE, IEEE, Seoul, South Korea, 577-582.

[32] Pyry Kettunen and J. Oksanen. 2018. Effects of Unsupervised Participation over the Internet on a Usability Study about Map Animation. In New Directions in Geovisual Analytics: Visualization, Computation, and Evaluation. Lipics, 02430 Finland, 7:1-7:7.

[33] Florian Keusch. 2015. Why do people participate in Web surveys? Applying survey participation theory to Internet survey data collection. Management review quarterly 65, 3 (2015), 183-216.

[34] Konstantina Kilteni, Ilias Bergstrom, and Mel Slater. 2013. Drumming in immersive virtual reality: the body shapes the way we play. IEEE transactions on visualization and computer graphics 19, 4 (2013), 597-605.

[35] Konstantina Kilteni, Raphaela Groten, and Mel Slater. 2012. The sense of embodiment in virtual reality. Presence: Teleoperators and Virtual Environments 21, 4 (2012), 373-387.

[36] Panagiotis Kourtesis, Danai Korre, Simona Collina, Leonidas AA Doumas, and Sarah E MacPherson. 2020. Guidelines for the development of immersive virtual reality software for cognitive neuroscience and neuropsychology: the development of virtual reality everyday assessment lab (VR-EAL), a neuropsychological test battery in immersive virtual reality. Frontiers in Computer Science 1 (2020), 12.

[37] John H Krantz and Reeshad Dalal. 2000. Validity of Web-based psychological research. In Psychological experiments on the Internet. Elsevier, Indiana 47243 , $35-60$.
[38] A. Lacroix, R. Kressig, T. Muehlbauer, Y. Gschwind, B. Pfenninger, O. Bruegger, and U. Granacher. 2015. Effects of a Supervised versus an Unsupervised Combined Balance and Strength Training Program on Balance and Muscle Power in Healthy Older Adults: A Randomized Controlled Trial. Gerontology 62 (2015), 275 - 288.

[39] Barbara L. Ludlow. 2015. Virtual Reality: Emerging Applications and Future Directions. Rural Special Education Quarterly 34, 3 (2015), 3-10. https://doi.org/ 10.1177/875687051503400302

[40] Xiao Ma, Megan Cackett, Leslie Park, Eric Chien, and Mor Naaman. 2018. Webbased VR experiments powered by the crowd. In Proceedings of the 2018 World Wide Web Conference. International World Wide Web Conferences Steering Committee, Republic and Canton of Geneva, CHE, 33-43.

[41] Guido Makransky, Thomas S Terkildsen, and Richard E Mayer. 2019. Adding immersive virtual reality to a science lab simulation causes more presence but less learning. Learning and Instruction 60 (2019), 225-236.

[42] Winter Mason and Siddharth Suri. 2012. Conducting behavioral research on Amazon's Mechanical Turk. Behavior research methods 44, 1 (2012), 1-23.

[43] Youngme Moon. 1998. The effects of distance in local versus remote humancomputer interaction. In Proceedings of the SIGCHI conference on Human factors in computing systems. ACM Press/Addison-Wesley Publishing Co., USA, 103-108.

[44] Masahiro Mori, Karl F MacDorman, and Norri Kageki. 2012. The uncanny valley [from the field]. IEEE Robotics \& Automation Magazine 19, 2 (2012), 98-100.

[45] Jason D Moss and Eric R Muth. 2011. Characteristics of head-mounted displays and their effects on simulator sickness. Human factors 53, 3 (2011), 308-319.

[46] Aske Mottelson and Kasper Hornbæk. 2017. Virtual reality studies outside the laboratory. In Proceedings of the $23 \mathrm{rd}$ acm symposium on virtual reality software and technology. Association for Computing Machinery, New York, NY, USA, $1-10$.

[47] Xueni Pan and Antonia F de C Hamilton. 2018. Why and how to use virtual reality to study human social interaction: The challenges of exploring a new research landscape. British fournal of Psychology 109, 3 (2018), 395-417.

[48] Gabriele Paolacci and Jesse Chandler. 2014. Inside the Turk: Understanding Mechanical Turk as a participant pool. Current Directions in Psychological Science 23, 3 (2014), 184-188.

[49] Gabriele Paolacci, Jesse Chandler, and Panagiotis G Ipeirotis. 2010. Running experiments on amazon mechanical turk. Fudgment and Decision making 5, 5 (2010), 411-419.

[50] Eyal Peer, Laura Brandimarte, Sonam Samat, and Alessandro Acquisti. 2017. Beyond the Turk: Alternative platforms for crowdsourcing behavioral research. fournal of Experimental Social Psychology 70 (2017), 153-163.

[51] Eyal Peer, Joachim Vosgerau, and Alessandro Acquisti. 2014. Reputation as a sufficient condition for data quality on Amazon Mechanical Turk. Behavior research methods 46, 4 (2014), 1023-1031.

[52] Thammathip Piumsomboon, Gun Lee, Robert W Lindeman, and Mark Billinghurst. 2017. Exploring natural eye-gaze-based interaction for immersive virtual reality. In 2017 IEEE Symposium on 3D User Interfaces (3DUI). IEEE, IEEE, Los Angeles, CA, USA, 36-39.

[53] Jennifer Preece. 2016. Citizen science: New research challenges for humancomputer interaction. International fournal of Human-Computer Interaction 32, 8 (2016), 585-612.

[54] Jaziar Radianti, Tim A Majchrzak, Jennifer Fromm, and Isabell Wohlgenannt. 2020. A systematic review of immersive virtual reality applications for higher education: Design elements, lessons learned, and research agenda. Computers \& Education 147 (2020), 103778.

[55] Jack Ratcliffe and Laurissa Tokarchuk. 2020. Evidence for embodied cognition in immersive virtual environments using a second language learning environment. In 2020 IEEE Conference on Games (CoG). IEEE, IEEE, London, UK, 1-8.

[56] Jack Ratcliffe and Laurissa Tokarchuk. 2020. Presence, Embodied Interaction and Motivation: Distinct Learning Phenomena in an Immersive Virtual Environment. In Proceedings of the 28th ACM International Conference on Multimedia. IEEE, London, UK, 1-8.

[57] Ulf-Dietrich Reips. 2000. The Web experiment method: Advantages, disadvantages, and solutions. In Psychological experiments on the Internet. Elsevier, $\mathrm{CH}-8032$ Zurich Switzerland, 89-117.

[58] Yvonne Rogers and Paul Marshall. 2017. Research in the Wild. Synthesis Lectures on Human-Centered Informatics 10, 3 (2017), i-97.

[59] Robert S. Ryan, Mara Wilde, and Samantha Crist. 2013. Compared to a small, supervised lab experiment, a large, unsupervised web-based experiment on a previously unknown effect has benefits that outweigh its potential costs. Computers in Human Behavior 29, 4 (2013), 1295 - 1301. https://doi.org/10.1016/j.chb. 2013.01.024

[60] David Saffo, Caglar Yildirim, Sara Di Bartolomeo, and Cody Dunne. 2020. Crowdsourcing Virtual Reality Experiments using VRChat. In Extended Abstracts of the 2020 CHI Conference on Human Factors in Computing Systems. Association for Computing Machinery, New York, NY, USA, 1-8.

[61] Ferran Argelaguet Sanz, Anne-Hélène Olivier, Gerd Bruder, Julien Pettré, and Anatole Lécuyer. 2015. Virtual proxemics: Locomotion in the presence of obstacles in large immersive projection environments. In 2015 IEEE Virtual Reality (VR). IEEE, IEEE, Arles, France, 75-80. 
[62] Valentin Schwind, Pascal Knierim, Nico Haas, and Niels Henze. 2019. Using presence questionnaires in virtual reality. In Proceedings of the 2019 CHI Conference on Human Factors in Computing Systems. Association for Computing Machinery, New York, NY, USA, 1-12.

[63] Kathryn Y Segovia and Jeremy N Bailenson. 2009. Virtually true: Children's acquisition of false memories in virtual reality. Media Psychology 12, 4 (2009), 371-393.

[64] C Senior, Mary L Phillips, J Barnes, and AS David. 1999. An investigation into the perception of dominance from schematic faces: A study using the World-Wide Web. Behavior Research Methods, Instruments, \& Computers 31, 2 (1999), 341-346.

[65] Francesco Soave, Nick Bryan-Kinns, and Ildar Farkhatdinov. 2020. A Preliminary Study on Full-Body Haptic Stimulation on Modulating Self-motion Perception in Virtual Reality. In Augmented Reality, Virtual Reality, and Computer Graphics, Lucio Tommaso De Paolis and Patrick Bourdot (Eds.). Springer International Publishing, Cham, 461-469.

[66] Jon Sprouse. 2011. A validation of Amazon Mechanical Turk for the collection of acceptability judgments in linguistic theory. Behavior research methods 43,1 (2011), 155-167.

[67] A. Steed, S. Frlston, M. M. Lopez, J. Drummond, Y. Pan, and D. Swapp. 2016. An 'In the Wild' Experiment on Presence and Embodiment using Consumer Virtual Reality Equipment. IEEE Transactions on Visualization and Computer Graphics 22, 4 (2016), 1406-1414.

[68] Anthony Steed, Francisco Ortega, Adam Williams, Ernst Kruijff, Wolfgang Stuerzlinger, Anil Ufuk Batmaz, Andrea Won, Evan Suma Rosenberg, Adalberto Simeone, Aleshia Hayes, and et al. 2020. Interactions. https://interactions.acm.org/blog/view/evaluating-immersiveexperiences-during-covid-19-and-beyond\#Ref2

[69] Anthony Steed, Ye Pan, Fiona Zisch, and William Steptoe. 2016. The impact of a self-avatar on cognitive load in immersive virtual reality. In 2016 IEEE Virtual Reality (VR). IEEE, IEEE, Greenville, SC, 67-76.

[70] Steven E Stern and Jon E Faber. 1997. The lost e-mail method: Milgram's lostletter technique in the age of the Internet. Behavior Research Methods, Instruments,
\& Computers 29, 2 (1997), 260-263.

[71] Ayoung Suh and Jane Prophet. 2018. The state of immersive technology research: A literature analysis. Computers in Human Behavior 86 (2018), 77-90.

[72] John Sweller. 2010. Cognitive load theory: Recent theoretical advances. Cognitive Load Theory: Recent Theoretical Advances 1, 2 (2010), 29-47.

[73] H Tankovska. 2020. AR/VR headset shipments worldwide 2020-2025. https://www.statista.com/statistics/653390/worldwide-virtual-and-augmentedreality-headset-shipments/

[74] Tom Tullis, Stan Fleischman, Michelle McNulty, Carrie Cianchette, and Margaret Bergel. 2002. An empirical comparison of lab and remote usability testing of web sites. In Usability Professionals Association Conference. Usability Professionals Association Conference, Orlando, FL, 8.

[75] David Waller, Eric Bachmann, Eric Hodgson, and Andrew C Beall. 2007. The HIVE: A huge immersive virtual environment for research in spatial cognition. Behavior Research Methods 39, 4 (2007), 835-843.

[76] Vivek R Warriar, John R Woodward, and Laurissa Tokarchuk. 2019. Modelling Player Preferences in AR Mobile Games. In 2019 IEEE Conference on Games (CoG). IEEE, IEEE, London UK, 1-8.

[77] Raphael P Weibel, Jascha Grübel, Hantao Zhao, Tyler Thrash, Dario Meloni, Christoph Hölscher, and Victor R Schinazi. 2018. Virtual reality experiments with physiological measures. FoVE (fournal of Visualized Experiments) 1, 138 (2018), e58318.

[78] Christopher J Wilson and Alessandro Soranzo. 2015. The use of virtual reality in psychology: a case study in visual perception. Computational and mathematical methods in medicine 2015 (2015), 7.

[79] Ye Yuan and Anthony Steed. 2010. Is the rubber hand illusion induced by immersive virtual reality? In 2010 IEEE Virtual Reality Conference (VR). IEEE, IEEE, Waltham, MA, USA, 95-102.

[80] Leshao Zhang and Patrick GT Healey. 2018. Human, Chameleon or Nodding Dog?. In Proceedings of the 20th ACM International Conference on Multimodal Interaction. Association for Computing Machinery, New York, NY, USA, 428-436. 